\title{
Philosophiques
}

\section{Critique et réflexion}

\section{La réflexion dans la Théorie critique de l'École de Francfort}

\section{Franck Fischbach}

Volume 43, numéro 2, automne 2016

Dossier. Usages de la réflexivité en philosophie allemande

URI : https://id.erudit.org/iderudit/1038205ar

DOI : https://doi.org/10.7202/1038205ar

Aller au sommaire du numéro

\section{Éditeur(s)}

Société de philosophie du Québec

ISSN

0316-2923 (imprimé)

1492-1391 (numérique)

Découvrir la revue

Citer cet article

Fischbach, F. (2016). Critique et réflexion : la réflexion dans la Théorie critique de l'École de Francfort. Philosophiques, 43(2), 233-248.

https://doi.org/10.7202/1038205ar
Résumé de l'article

Le concept de réflexion joue un rôle clé dans la constitution de la théorie critique dès les premiers textes de Horkheimer dans les années 30 . Mais la théorie critique n'a pas le monopole de la réflexion et la théorie traditionnelle la met également en oeuvre : c'est donc qu'il y a une spécificité de la réflexion engagée par la théorie critique. Au fil d'une enquête qui va de Horkheimer à Habermas, le présent article tente de localiser cette spécificité dans la particularité d'une expérience qui conduit à la réflexion, à l'adoption de l'attitude critique et ouvre l'horizon de l'émancipation. 


\title{
Critique et réflexion
}

\section{La réflexion dans la Théorie critique de l'École de Francfort FRANCK FISCHBACH}

Université de Strasbourg / CREPHAC EA 2326

\begin{abstract}
RÉSUMÉ - Le concept de réflexion joue un rôle clé dans la constitution de la théorie critique dès les premiers textes de Horkheimer dans les années 30. Mais la théorie critique n'a pas le monopole de la réflexion et la théorie traditionnelle la met également en œuvre: c'est donc qu'il y a une spécificité de la réflexion engagée par la théorie critique. Au fil d'une enquête qui va de Horkheimer à Habermas, le présent article tente de localiser cette spécificité dans la particularité d'une expérience qui conduit à la réflexion, à l'adoption de l'attitude critique et ouvre l'horizon de l'émancipation.
\end{abstract}

\begin{abstract}
The concept of reflection plays an important part in the critical theory right from the beginning of the Frankfurter School in Horkheimer's texts during the 30s. However, the critical theory doesn't have a monopoly on reflection and the traditional theory resorts also to it. Therefore, the critical use of the reflection must have a specificity that the present article aims at bringing to light. Following a path from Horkheimer to Habermas, this article tries to set this specificity in an individual and collective experience that can lead to reflection, to the adoption of the critical attitude and opens the perspective of emancipation.
\end{abstract}

Dès les textes fondateurs des années 30 , la théorie critique se présente et se comprend elle-même comme la mise en œuvre d'une démarche de type essentiellement réflexif. Il n'y a de théorie critique possible ou de théorie possible comme critique que grâce à la réflexion. Le propre de cette réflexion est de produire ce que Horkheimer appelait une "clarification» (Klärung). Encore cet effet de clarification provoqué par la réflexion n'a-t-il rien de spécifique à la théorie de type critique: une réflexion provoquant une clarification existe bien sûr aussi dans la théorie de type traditionnel. Horkheimer le mentionne d'ailleurs lui-même:

la pensée bourgeoise est ainsi faite que, dans la réflexion ${ }^{1}$ sur son propre sujet (Subjekt), elle reconnaît de façon logiquement nécessaire l'ego qui s'imagine être autonome; cette pensée est par essence abstraite et son principe est l'indi-

1. Notons que dans la version du texte initialement parue en I937 dans la Zeitschrift für Sozialforschung, le terme utilisé était "die Rückwendung" (que l'on peut rendre par "retour sur soi »); c'est dans la réédition de I968 que Horkheimer a remplacé « die Rückwendung » par "die Reflexion ». On peut voir là, de la part de Horkheimer, une volonté de rendre plus explicite et plus immédiatement identifiable le concept philosophique de réflexion que le terme de Rückwendung dissimulait ou rendait inapparent. 
vidualité coupée de tout devenir, l'individualité qui se prend pour la cause première du monde, voire carrément pour le monde même ${ }^{2}$.

On a là le produit de la clarification engendré par la théorie traditionnelle quand elle réfléchit au sujet qui est le sien et tente de le saisir réflexivement: le produit de cette clarification réflexive est dans ce cas l'ego comme sujet à la fois individuel et universel (universel dans son individualité, c'est-à-dire dans son isolement) de la pensée ou de la connaissance, c'est-à-dire l'ego comme sujet abstrait de la pensée. Abstrait, ce sujet tel que mis au jour par la réflexion de la théorie traditionnelle l'est parce qu'il est considéré abstraction faite de "ses rapports réels avec d'autres individus et avec des groupes, de sa confrontation avec une classe déterminée », et indépendamment de "son insertion médiatisée dans l'ensemble de la société et dans la nature » ${ }^{3}$. La clarification réflexive n'est donc pas propre en elle-même à la seule théorie critique, de sorte que la clarification qui est propre à la réflexion engagée par le théoricien critique doit posséder quelque chose de particulier et de spécifique qui la distingue de la réflexion telle qu'elle est pratiquée dans le cadre d'une théorie de type traditionnel.

Cette spécificité est ainsi décrite par Horkheimer:

dans une pensée critique véritable, la clarification ne possède pas seulement le sens d'un processus logique, mais tout autant celui d'un processus historique concret; dans le cours de ce processus, ce qui change, c'est aussi bien la structure sociale dans son ensemble que la relation du théoricien à la société en général, autrement dit: ce qui change, c'est le sujet autant que le rôle de la pensée ${ }^{4}$.

De cette proposition, on peut déjà déduire que la réflexion, dans le cadre d'une théorie de type critique, produit une clarification qui a ceci de spécifique qu'elle possède une dimension expérientielle. Cela signifie que la clarification réflexive propre à la théorie critique n'a pas lieu pour la pensée seulement, dans le seul élément de la pensée ou, comme le dit Horkheimer, qu'elle n'est pas seulement un "processus logique». La clarification réflexive de type critique produit des effets, en l'occurrence des effets de transformation, à la fois sur le sujet même de la réflexion et sur les rapports que le sujet de la réflexion entretient avec le monde qui est le sien. Le sujet expérimente donc une transformation, un changement de ses rapports au monde et une modification de lui-même comme accompagnant ou résultant de la mise en œuvre de la réflexion quand celle-ci est menée d'un point de vue critique.

2. Max Horkheimer, «Traditionelle und kritische Theorie» (I937), in: Traditionelle und kritische Theorie, Fünf Aufsätze, Frankfurt a. M., Fischer Taschenbuch Verlag, 20II, p. 227 [noté: TKT]; Théorie traditionnelle et théorie critique, trad. C. Maillard et S. Muller, Paris, Gallimard, I974, p. 42 (noté: TTTC; traduction modifiée par nous, F. F.).

3. TKT, p. 227; TTTC, p. 43 (trad. modifiée).

4. TKT, p. 228; TTTC, p. 43 (trad. modifiée). 
Pour le dire autrement, la réflexion clarifiante ou la clarification réflexive, quand elles sont critiques, ont pour effet de modifier ou transformer à la fois le rapport sujet/objet et le sujet lui-même. Un exemple intéressant et utile est donné par Horkheimer quand il entreprend de distinguer entre la théorie critique et une autre démarche intellectuelle qui peut paraître en être relativement proche et avec laquelle il serait aisé de la confondre: la théorie critique reposant sur "une attitude [ein Verhalten] qui consiste à prendre pour objet la société elle-même ${ }^{5}$ » et à considérer «le travail productif sur le plan de la théorie ${ }^{6} »$ comme une activité sociale parmi les autres qui résulte comme les autres de la division sociale du travail et qui fait l'objet, comme toute activité utile, d'une demande sociale ${ }^{7}-$ la théorie critique donc, parce qu'elle approche le travail théorique de cette façon, peut être aisément confondue avec une démarche de type sociologique qui prendrait pour objet d'analyse «le caractère socialement conditionné des théories $^{8} »$. Horkheimer explique que ce serait là une erreur dans la mesure où une enquête portant sur les déterminismes et les conditionnements sociaux qui pèsent sur les théories scientifiques et philosophiques relève elle-même encore complètement de la théorie traditionnelle:

l'étude de l'idéologie ou la sociologie du savoir, que l'on a extraites en dehors de la théorie critique de la société et que l'on a établies comme des disciplines particulières, ne se trouvent en contradiction ni selon leur essence ni d'après leur ambition avec la manière courante de procéder propre à la science qui ordonne des faits?

On a en réalité affaire ici à une démarche typique de la théorie traditionnelle qui, d'une part, connecte des faits ou des ordres de faits les uns aux autres (en vue de mettre au jour des connexions régulières) et qui, d'autre part, considère les faits en questions comme extérieurs au théoricien et indépendants du scientifique.

Il ne s'agit certes pas de nier que, dans ces tentatives de connecter l'ordre des idées et des représentations avec celui des processus sociaux, et de mettre au jour les conditionnements sociaux qui pèsent sur la formation des idées et des représentations, il y ait la mise en œuvre d'une forme de réflexion: simplement, "la connaissance de soi de la pensée est ici réduite au fait de dévoiler les relations entre les positions intellectuelles qu'on adopte et le lieu social qu'on occupe ${ }^{10}$ ». Or, ajoute Horkheimer, «la structure de l'attitude critique - dont les intentions s'étendent au delà de la praxis sociale régnante - n'est certainement pas plus apparentée à de telles disci-
5. TKT, p. 223; TTTC, p. 38 .
6. Ibid., p. 37 .
7. Ibid.
8. TKT, p. 225 ; TTTC, p. 40 (trad. modifiée).
9. TKT, p. 226; TTTC, p. 40-4I (trad. modifiée).
10. TKT, p. 226; TTTC, p. 4I (trad. modifiée). 
plines sociales qu'à la science de la nature ${ }^{11}$ ». Et si elle ne l'est pas, c'est pour la raison suivante: "l'opposition [de l'attitude critique] au concept traditionnel de la théorie ne provient de façon générale pas tant d'une différence des objets que d'une différence des sujets ${ }^{12}{ }^{»}$. En d'autres termes, il ne suffit pas, pour qu'une réflexion soit critique et relève de la théorie critique, qu'elle porte sur des objets sociaux, et ce n'est pas parce qu'une réflexion portera sur le contexte social de formation des idées et des représentations qu'elle sera pour autant une réflexion critique: ce n'est pas l'objet de la réflexion qui compte, c'est son sujet. Et le propre de la réflexion critique est de reposer sur une transformation de l'attitude du sujet réfléchissant, sur une modification de son attitude qui ne peut elle-même s'expliquer que par une certaine expérience qui est effectivement faite par le sujet. La question est de savoir quelle est la nature de cette expérience.

Lorsque Horkheimer écrit que «la confiance dans le fil conducteur que la vie sociale, telle qu'elle se déroule maintenant, met dans la main de chacun fait complètement défaut à l'attitude critique ${ }^{13}{ }^{\prime}$, il faut comprendre que ce manque complet de confiance repose sur une expérience faite par le sujet qui adopte cette attitude critique: le résultat de cette expérience faite par l'individu qui adopte l'attitude critique est qu'il ne se comporte plus de la même manière et, notamment,

qu'il [n']accepte [plus] comme données les déterminations fondamentales de son existence et [n']aspire [plus] à les satisfaire, [ne] trouve [plus] sa satisfaction et son honneur dans le fait d'accomplir autant qu'il le peut les devoirs liés à la place qu'il occupe dans la société, et [n']accomplit [plus] consciencieusement son devoir avec toute l'énergie critique qui peut être mise dans le détail ${ }^{14}$.

Mais en quoi consiste véritablement l'expérience qui produit subjectivement un tel résultat, et pourquoi conduit-elle à une telle défiance envers la société telle qu'elle est? Cette expérience est fondamentalement celle du "caractère scindé de la totalité sociale dans sa forme actuelle ${ }^{15}$ ", ou plutôt : l'expérience consiste en ce que ce "caractère scindé de la totalité sociale dans sa forme actuelle se développe en une contradiction consciente chez les sujets de l'attitude critique ${ }^{16} »$.

Les sujets de l'attitude critique (ou de la "conduite» critique, Verhalten) font donc l'épreuve ou l'expérience d'une scission de la société qui accède chez eux au niveau de la conscience d'une contradiction. Les termes de cette contradiction peuvent être décrits de la façon suivante. D'un côté les sujets en passe d'adopter l'attitude critique «reconnaissent l'organi-

11. Ibid.; ibid. (trad. modifiée).

12. Ibid.; ibid. (trad. modifiée).

13. TKT, p. 224; TTTC, p. 38 (trad. modifiée).

14. Ibid.; ibid., p. 38 (trad. modifiée).

15. Ibid.; ibid., ibid.

16. Ibid.; TTTC, p. 39 (trad. modifiée). 
sation présente de l'économie et l'ensemble de la culture qui est fondée sur elle comme les produits du travail humain, comme l'organisation dont l'humanité s'est dotée et dont elle était capable à cette époque ${ }^{17} »$. De ce point de vue là, parce qu'ils comprennent l'organisation sociale existante comme celle que le travail humain a engendrée et produite, les sujets peuvent s'identifier à cette organisation dès lors qu'ils la reconnaissent comme étant aussi leur propre produit. Les sujets reconnaissent alors l'organisation sociale existante comme le résultat et le produit «de la volonté et de la raison» et donc comme étant "leur propre monde ${ }^{18}$ " dans la mesure où, comme le précise Horkheimer, "l'œuvrer-ensemble (das Zusammenwirken ${ }^{19}$ ) des hommes dans la société est le mode d'existence de leur raison ${ }^{20}$ ". Mais, d'un autre côté, les mêmes sujets font en même temps «l'expérience de ce que la société est comparable aux processus naturels extrahumains et à de purs et simples mécanismes parce que les formes de culture reposant sur la lutte et l'oppression ne portent pas témoignage d'une volonté unifiée et consciente d'elle-même $\mathrm{e}^{21} »$. C'est bien toujours du même monde social que précédemment qu'il s'agit, compris cette fois par les sujets comme «un monde qui n'est pas le leur, mais qui est celui du capital » : cette seconde compréhension du même monde est directement contradictoire avec la précédente. L'expérience qui engendre l'adoption de l'attitude critique est l'expérience même de cette contradiction entre un monde social compris comme devant être rationnel puisqu'il résulte du travail et de l'activité collective des hommes, et un monde social effectivement existant compris cette fois comme un monde échappant à l'emprise de la raison et de la volonté des hommes et s'imposant à eux avec la contrainte et la nécessité d'un monde objectif et naturel. C'est l'expérience vive qu'il fait de cette contradiction qui modifie à la fois le sujet et son rapport au monde: la modification du sujet consiste en l'adoption de la posture de réflexion critique, tandis que la modification de son rapport au monde consiste en ce qu'il considère désormais le monde social comme un monde devant être transformé.

On a affaire là à une transformation qui possède à la fois, du côté du sujet, une dimension théorique ou épistémologique et, du côté cette fois de l'objet (ou du monde), une dimension pratique - ces deux dimensions devenant absolument indissociables l'une de l'autre. Du point de vue pratique, "l'attitude critique a pour but la transformation du tout [social] $]^{22}$ » : il s'agit de faire en sorte que les acteurs sociaux fassent du monde social leur monde, qu'ils le soumettent à leur volonté rationnelle et cessent de se le laisser

17. Ibid.; ibid. (trad. modifiée).

18. Ibid.; ibid. (trad. modifiée).

19. Das Zusammenwirken: un terme que l'on peut aussi traduire quasi littéralement par «la co-opération».

20. TKT, p. 220; TTTC, p. 34 (trad. modifiée).

21. TKT, p. 224; TTTC, p. 39 (trad. modifiée).

22 TKT, p. 225 ; TTTC, p. 40 (trad. modifiée). 
imposer de facto comme une réalité donnée et naturelle ou quasi-naturelle échappant à leur maîtrise. Il s'agit que les acteurs sociaux aient la maîtrise de leur propre vie collective et sociale comme ils ont, dans la modernité, la maîtrise de leur vie individuelle - la contradiction dont le sujet critique fait l'expérience étant précisément celle entre le niveau individuel de l'existence, où la maîtrise rationnelle et volontaire est possible, et le niveau social ou collectif où une maîtrise comparable apparaît au contraire comme impossible et hors de portée. C'est là, à proprement parler, d'un projet d'émancipation qu'il s'agit, un projet dont Horkheimer précise la nature en disant qu'il est celui d'une "nouvelle organisation du travail ${ }^{23}$ ".

Il s'agit en effet de mettre fin à une organisation du travail qui n'a d'organisation que le nom puisqu'elle relève en réalité d'une division spontanée et naturelle (donc, précisément, non organisée) du travail que les acteurs ne maîtrisent pas et qui s'impose à eux. Aussi Horkheimer peut-il écrire que "la pensée critique est motivée aujourd'hui par la tentative de réellement dépasser la scission, de supprimer la contradiction entre la conscience des buts, la spontanéité et la rationalité telles qu'elles existent au niveau de l'individu, et les rapports liés au procès de travail et qui fondent la sociétée ${ }^{4}$ ». Les caractéristiques qui prévalent au niveau de l'individu (la conscience des buts, la spontanéité de la volonté, le caractère raisonnable de cette volonté et de ses buts) ne doivent pas disparaître et s'effacer pour laisser place à leurs contraires (buts inconscients, contrainte et irrationalité) dès qu'on passe au niveau collectif et social, dès qu'il ne s'agit plus de l'agir individuel, mais des interactions sociales entre les individus en tant qu'ils travaillent, produisent et échangent.

Toutefois, la transformation du sujet et de son rapport au monde impliquée par l'adoption de l'attitude de réflexion critique possède également une dimension théorique ou épistémologique. Cette transformation consiste essentiellement en ce que les objets de la pensée, de la connaissance et de l'enquête ne peuvent plus être considérés par le sujet comme de purs et simples faits. La théorie de type critique est conçue par Horkheimer comme "une démarche théorique qui ne consiste pas en dernière analyse à déterminer des faits à partir de systèmes de concepts aussi simples et différenciés que possible ${ }^{25}$ ». C'est que, au regard de celui qui a fait l'expérience de la contradiction précédemment décrite, et que sa réflexion sur cette expérience a conduit à l'adoption de l'attitude critique, au regard de celui-là donc, il n'y a plus à proprement parler de faits, si l'on entend par là des données simplement trouvées et considérées comme existant objectivement en dehors et indépendamment du sujet. Horkheimer écrit:

23 TKT, p. 226; TTTC, p. 4I.

24 Ibid.; ibid. (trad. modifiée).

25. TKT, p. 225 ; TTTC, p. 40 (trad. modifée). 
Dans la mesure où les contenus de choses (die Sachverhalte), qui sont donnés dans la perception, sont conçus comme des produits qui relèvent fondamentalement du contrôle humain et qui doivent de toute façon en relever à l'avenir, ils perdent leur caractère de simple facticité (Tatsächlichkeit) ${ }^{26}$.

Les produits se substituent aux faits, il n'y a plus de donnés, il n'y a plus que des résultats toujours susceptibles d'être repris et réélaborés par la même activité humaine qui les a initialement engendrés. Ou, autrement dit, il n’y a pas d'objet qui soit de toute éternité et définitivement ce qu'il est: en tant qu'objet, il est à chaque fois l'objet d'un sujet qui peut le modifier, le transformer pour en faire un autre et nouvel objet. À quoi il faut encore ajouter que les objets ne sont pas les produits de l'activité d'un sujet individuel et isolé, mais toujours ceux d'une activité sociale faite de la combinaison des activités individuelles. Et c'est pourquoi le théoricien critique est directement opposé au "spécialiste qui, en tant que scientifique, considère la réalité sociale et l'ensemble de ses produits comme extérieurs [à lui] ${ }^{27}$ ». Les «objets» du théoricien critique sont des réalités socialement et historiquement engendrées qui ne sont rien d'extérieur pour lui puisqu'ils sont les produits d'une activité sociale dont il sait que sa propre réflexion théorique est elle-même une composante et une expression.

On objectera certainement qu'il existe au moins les objets et réalités qu'on appelle naturels en ce que précisément ils ne sont pas les produits de l'activité humaine et qu'ils précèdent au contraire celle-ci. Horkheimer admet volontiers qu'il "subsistera toujours quelque chose d'extérieur à l'activité intellectuelle et matérielle des hommes ${ }^{28}$ ", et que c'est précisément ce "quelque chose» qu'on appelle "la nature». Mais il ne faut pas entendre par là quoi que ce soit qui serait radicalement et encore moins définitivement extérieur aux hommes et à leurs activités: au contraire, ce qui est extérieur à l'activité humaine varie considérablement selon les sociétés et selon les époques de l'histoire. Cette réalité extérieure à l'activité humaine qu'on appelle "la nature» est donc toute relative et elle ne consiste finalement en rien d'autre qu'en «l'ensemble des facteurs qui, à chaque fois, ne sont pas encore maîtrisés et auxquels la société a affaire ${ }^{29} »$. La nature n'est en ce sens rien de véritablement ni de définitivement extérieur à l'activité humaine, elle est l'ensemble variable, changeant et modifiable de ce qui, à un stade donné du développement de la connaissance et de la technique, reste à ce stade encore à devoir être maîtrisé par l'activité matérielle et intellectuelle que les hommes déploient dans leurs sociétés.

Ce qui nous conduit à un autre trait caractéristique de la théorie critique et de l'attitude de réflexion critique qui la sous-tend et dont on a vu

26. TKT, p. 226; TTTC, p. 226 (trad. modifiée).

27. Ibid.; ibid. (trad. modifiée).

28. TKT, p. 227 ; TTTC, p. 42 (trad. modifiée).

29. Ibid.; ibid. (trad. modifiée). 
qu'elle provenait de l'expérience faite d'une contradiction: de même que le lieu de l'épreuve ou de l'expérience de cette contradiction est historiquement situé (il s'agit des sociétés modernes comme lieux de la contradiction entre le caractère raisonnable et maîtrisé de l'existence individuelle et le caractère déraisonnable et non maîtrisé des interactions collectives et de la vie sociale en général), de même la réflexion critique engage-t-elle un rapport à l'histoire. On vient déjà de le voir dans la conception que Horkheimer se fait de la nature: dire que la nature n'est que l'ensemble des facteurs non encore maîtrisés par l'activité humaine, c'est dire que l'histoire est fondamentalement de développement des capacités humaines pratiques et théoriques de maîtrise de la nature. Or de telles capacités ne pouvant se développer que socialement, cela revient donc à dire que l'histoire est fondamentalement le processus progressif de maîtrise théorique et pratique des forces naturelles par les sociétés humaines.

Cependant, la chose est plus apparente encore dans les vues que Horkheimer propose au sujet du développement des sociétés humaines considérées cette fois en elles-mêmes et indépendamment de leur rapport à la nature. C'est là un point très clairement souligné par Axel Honneth:

dans son article programmatique de 1937 sur la distinction entre théorie traditionnelle et théorie critique, Horkheimer dégageait déjà clairement le principe méthodologique qui allait désormais guider le travail de l'Institut [;] puisque la Théorie critique, à la différence des approches traditionnelles, devait avoir conscience à la fois de son enracinement social et du contexte de sa mise en œuvre politique, puisqu'elle constituait une sorte d'autoréflexion du processus historique, les normes et les principes mobilisés devaient tous être ancrés en quelque manière dans la réalité historique ${ }^{30}$.

Or nous avons déjà rencontré ces "normes et principes " : il s'agit en particulier de l'idée normative d'un ordre social conforme à la raison qui soit susceptible d'être à la fois voulu et maîtrisé par les acteurs sociaux. Et cette idée est considérée par Horkheimer comme possédant un ancrage dans la réalité sociale elle-même: la raison, selon lui, existe déjà, et elle est à l'œuvre dans la réalité sociale. Ce qui est le sens même de la proposition fondamentale déjà citée: «la coopération (das Zusammenwirken) des hommes dans la société est le mode d'existence de leur raison ${ }^{31} »$. De cette proposition fondamentale il découle une idée de ce que doit être la fin ou le but visé par le processus historique de développement des sociétés humaines: à savoir des sociétés de plus en plus conformes à la raison grâce à la maximisation en elle de l'agir coopératif. Horkheimer le dit clairement:

30. Axel Honneth, «Une critique reconstructive de la société sous réserve généalogique ", in: A. Honneth, Ce que social veut dire, tome 2: Les pathologies de la raison, trad. P. Rusch, Paris, Gallimard, 201 5, p. 89 (c'est nous qui soulignons).

31. TKT, p. 220; TTTC, p. 34 (trad. modifiée). 
les points de vue que la théorie critique tire de l'analyse historique comme étant les buts de l'activité humaine - avant tout l'idée d'une organisation sociale conforme à la raison et à l'intérêt commun à tous — sont immanents au travail humain, sans que les individus ou l'esprit public en ait conscience sous une forme exacte; il faut un intérêt déterminé pour faire l'expérience de ces tendances et les percevoir ${ }^{32}$.

Cet intérêt est directement lié à l'expérience de la contradiction dont on a vu qu'elle provoque l'adoption du point de vue réflexif et critique: cette expérience engendre un intérêt à sortir de la dite contradiction, un intérêt à s'en extraire en y mettant un terme, c'est-à-dire en lui trouvant une solution. Cet intérêt pour la sortie de la contradiction et pour sa résolution est en même temps un intérêt qui porte à mettre un terme à une expérience qui est essentiellement négative et qui consiste en l'épreuve faite d'une souffrance tant individuelle que collective. Le lien entre l'épreuve de cette souffrance et l'expérience de la contradiction est au demeurant clairement établi par Horkheimer :

la manière bourgeoise dont fonctionne l'économie n'est maîtrisée par aucun plan que puissent apercevoir les individus en concurrence, quelle que soit la perspicacité dont ils fassent preuve, elle n'est pas orientée de façon consciente vers un but; la vie du tout qui provient d'elle ne se réalise, au prix d'énormes frictions, que sous une forme atrophiée et, en même temps, soumise au hasard ${ }^{33}$.

C'est bien de cela qu'il s'agit de s'extraire: d'une forme sociale de vie atrophiée qui n'offre aux individus aucune perspective de réalisation d'euxmêmes et qui soumet leur existence au règne du hasard et de la contingence. Il s'agit donc de l'intérêt qui porte ceux qui font l'expérience négative d'une souffrance à sortir de cet état de souffrance et à se libérer de ce qui, dans l'état social existant, provoque et engendre cette souffrance: en un mot, il s'agit d'un intérêt à l'émancipation.

C'est là très exactement le point où Jürgen Habermas a repris et relancé l'analyse de la réflexion dans son lien à la fois à la tâche de clarification (Klärung) ou d'éclaircissement (Aufklärung), et à l'intérêt pour l'émancipation. Notons que Habermas fait cela alors que, dans l'histoire de la théorie critique telle qu'elle s'est poursuivie après les textes fondateurs publiés dans les années 30 par Horkheimer, dans l'exil américain puis en République fédérale après 45 , le motif de la réflexion avait eu tendance à s'effacer, tandis qu'en même temps le lien entre la réflexion, la critique et l'intérêt à l'émancipation devenait de plus en plus ténu. Chez Adorno, par exemple, la réflexion critique n'aboutit guère plus qu'à la prise de conscience de "la discordance entre la puissance de l'organisation et celle de l'individu sin-

32. TKT, p. 230; TTTC, p. 45 (trad. modifiée).

33. TKT, p. 220; TTTC, p. 34 (trad. modifiée). 
gulier [...], entre la violence de ce qui est et l'impuissance de la pensée qui essaie de le pénétrer ${ }^{34} »$. Cette prise de conscience s'accompagne du renoncement à toute hypothèse relative à "l'existence d'un sujet commun pour ce qui concerne la configuration active et consciente de la société ", hypothèse qui reviendrait à «ignorer que l'essence [de cette société] consiste justement en l'absence de pareil sujet univoque de la raison ${ }^{35} »$. On voit clairement ici toute la distance prise à l'égard de ce qui était encore une conviction fondamentale chez Horkheimer, du moins dans les années 30 , à savoir la conviction d'origine hégélienne selon laquelle les échanges réciproques et les interactions sociales sont les lieux d'effectuation de la raison, une effectuation certes largement contrariée dans les conditions actuelles, mais qui constitue néanmoins le point d'appui pour l'exigence d'une effectuation plus complète, plus accomplie de cette même rationalité. On est très loin, chez Adorno, de perspectives de ce genre et, si l'autoréflexion critique n'est pas complètement abandonnée, elle n'aboutit plus qu'à produire une conscience lucide et désabusée de la situation réelle de l'individu et de sa complète impuissance: la réflexion critique ne peut guère plus qu'aboutir à ce que "l'individu singulier impuissant demeure malgré tout maître de lui-même à travers la conscience de sa propre impuissance ${ }^{36} »$.

Contre cette version pessimiste de la critique, l'assistant d'Adorno, Habermas, tente de renouer, dans Connaissance et intérêt (I968), le fil rompu qui reliait entre elles la réflexion, la critique et l'émancipation. Et il le fait en cherchant à articuler la théorie critique de la société avec la psychanalyse, ou plutôt à les réarticuler l'une à l'autre d'une nouvelle manière, tant les tentatives de le faire avaient déjà été nombreuses dès la première génération des théoriciens de Francfort (chez Horkheimer lui-même, mais aussi chez Fromm, Adorno et Marcuse). Les caractéristiques principales de l'expérience qui a pour effet de conduire le sujet à la réflexion et à l'adoption de l'attitude critique, ces caractéristiques que Horkheimer avait su mettre au jour, se retrouvent dans la description que donne Habermas de l'expérience qui pousse un sujet à entreprendre une cure analytique. Habermas commence par insister sur le fait que le sujet qui s'engage dans une analyse est d'abord un sujet souffrant: "au commencement, il y a l'expérience de la souffrance et de la détresse, et l'intérêt qu'on a à faire cesser cette condition accablante ${ }^{37} »$. C'est l'expérience de cette souffrance et le besoin, éprouvé par le sujet, de sortir de cette situation de détresse, de cette « condition acca-

34. Theodor W. Adorno, «Individu et organisation» (I953), in: Th. W. Adorno, Société: Intégration, Désintégration. Ecrits sociologiques, trad. P. Arnoux et alii, Paris, Payot, $20 I$ I, p. I75.

35. Ibid.

36. Ibid.

37. Jürgen Habermas, Connaissance et intérêt, trad. G. Clémençon et J.-M. Brohm, Paris, Gallimard, I976, p. 266 [noté: CI]; Jürgen Habermas, Erkenntnis und Interesse, Frankfurt a.M., Suhrkamp, STW, I973, 2003, p. 286 [noté: EI]. 
blante » qui, à eux deux, donnent naissance, dans le sujet, à la fois à l'attitude critique et à un "intérêt pour la connaissance de $\operatorname{soi}^{38}$ ». Mais comment comprendre que l'expérience que le sujet fait d'une souffrance puisse en même temps le conduire à adopter, vis-à-vis de lui-même, une attitude critique et réflexive? C'est que l'expérience qu'il fait est celle d'une autoaliénation ou d'une aliénation de lui-même, au sens précis où le sujet éprouve que quelque chose ou une part de lui-même lui est étrangère et ne lui est pas accessible. Comme le dit Axel Honneth, l'attitude réflexive permet au sujet de "se retourner sur les fragments détachés de l'histoire de sa vie, [et de] s'engager ainsi sur la voie du souvenir et [pour] finalement s'approprier après coup ce qui s'était détaché ${ }^{39}$ ". La situation du sujet est telle que sa propre identité ne lui est pas transparente, ou que quelque chose d'elle s'est séparé et prend la forme d'une réalité objective et impénétrable: il y a "critique» parce qu'il s'agit de dissoudre cet élément d'opacité objective au cœur du sujet. La critique au sens de la dissolution de ce point à la fois d'opacité, de résistance et d'étrangèreté dans le sujet est inséparable de la réflexion: "on peut appeler réflexion, écrit Habermas, le fait de rendre conscient ce qui est inconscient ${ }^{40} »$. Or ce qui est inconscient est précisément cette part de sa propre biographie à laquelle le sujet souffre de ne plus avoir accès, et c'est pourquoi la dissolution à la fois critique et analytique des obstacles qui empêchent le sujet d'accéder à cette part de sa biographie est en même temps la restitution synthétique de l'unité de cette même biographie: «la réalisation spécifique de l'autoréflexion» est telle qu'en elle "la dissolution analytique [et critique, F. F.] comme telle est la synthèse, le rétablissement d'une unité corrompue ${ }^{41}$ ».

On peut exprimer la même chose un peu différemment et dire que ce dont souffre le sujet est une forme d'incompréhension de lui-même au sens où une partie de sa propre vie est écrite dans un texte qu'il ne peut plus déchiffrer et encore moins comprendre. Le sujet souffre d'une rupture de la communication avec lui-même pour la raison que les symboles qui pourraient lui permettre d'interpréter ses propres désirs réprimés sont, d'une part, les symboles propres à un langage privé dont le sujet lui-même ne maîtrise plus les termes et, d'autre part, des symboles qui sont exclus de la communication publique: en conséquence, «la communication avec luimême du sujet parlant et agissant est interrompue ${ }^{42}$ ». D'où le rôle et la fonction qui sont ceux de l'analyste: il joue le rôle d'un interprète dont la fonction est d'apprendre au sujet à comprendre sa propre langue de sorte

38. CI, p. 267 ; EI, p. 287.

39. Axel Honneth, "S'approprier sa liberté. La conception freudienne de la relation individuelle à soi ", in: A. Honneth, Ce que social veut dire, tome 2: Les pathologies de la raison, trad. P. Rusch, Paris, Gallimard, 20I 5, p. 265.

40. J. Habermas, CI, p. 274; EI, p. 296.

41. CI, p. 266; EI, p. 286.

42. CI, p. 260; EI, p. 278. 
que, «instruit par l'analyste, le patient apprenne à lire ses propres textes, qu'il a lui-même mutilés et déformés, et à traduire dans le discours de la communication publique les symboles d'un discours déformé en langage privé $^{43}$ ». Ce processus de traduction est en lui-même la mise en œuvre d'une réflexion, et "l'acte de compréhension auquel mène [cette dernière] est une autoréflexion ${ }^{44}$ ». Le fait, comme disait Freud, de «rendre l'inconscient accessible au conscient» est en effet un processus de réflexion en ce qu'il «n'est pas seulement un processus sur un plan cognitif, mais est en même temps la dissolution des résistances sur un plan affectif ${ }^{45}$ » : en d'autres termes, la cure est un processus de réflexion au sens fort du terme précisément parce que c'est en même temps un processus critique, c'est-à-dire un processus qui agit par la dissolution d'obstacles et de résistances dont le résultat est une modification réelle et qualitative du sujet. Ce n'est pas seulement que le sujet apprend des choses sur lui-même (ce qui serait un processus seulement cognitif), c'est que, les apprenant, il change et se transforme. Cette transformation consiste essentiellement en ce que le sujet trouve au terme de la cure un accès à soi comme sujet complet auquel n'échappe plus des pans entiers de lui-même. C'est là ce qui permet à Habermas d'écrire que «la critique s'achève dans une transformation de la base affective motivationnelle, de même qu'elle commence par le besoin d'une transformation pratique ${ }^{46}$ ». C'est bien en vertu de ce lien avec la critique que la réflexion n'est pas un processus seulement ou purement cognitif: elle ne serait que cela s'il n'y avait pas d'abord et dès le commencement, du côté du sujet, l'expérience d'un besoin de changement (qui est, on l'a vu, le besoin de s'extraire d'un état ressenti comme un état de souffrance) et, à l'arrivée, l'expérience par le sujet d'un état qualitativement nouveau par rapport à l'état de départ. La critique est liée au besoin de dissoudre des obstacles et des résistances et au besoin de parvenir, grâce à cette dissolution, à un état qualitativement autre et nouveau. En d'autres termes, la critique est liée au besoin de se délivrer, de se libérer d'un état de souffrance et de l'influence des causes qui le provoquent.

Si l'expérience d'une souffrance et le besoin de guérir sont ce qui conduit un patient aussi bien chez un médecin que chez un analyste, il y a cependant dans le second cas une spécificité qui est directement liée au rôle que joue la critique dans le cas de l'analyse: "à la différence du traitement médical habituel, la pression de la souffrance et l'intérêt qu'on a à guérir ne sont pas seulement [dans le cas de l'analyse] l'occasion qui détermine le commencement de la thérapeutique, mais la condition préalable de son succès $^{47}{ }^{7}$. Cela signifie que, dans le cas de l'analyse, l'expérience de la souf-

43. CI, p. 26I ; EI, p. 280.

44. CI, p. 26I; EI, p. 280.

45. CI, p. 262; EI, p. 28 I.

46. CI, p. 266; EI, p. 286.

47. CI, p. 266; EI, p. 286-287. 
france et l'intérêt à la guérison jouent un rôle non seulement au départ dans la décision d'aller consulter, mais continuent à jouer un rôle décisif dans la poursuite de la cure, au point que Freud allait jusqu'à dire du psychanalyste qu'il devait "veiller à ce que les souffrances du malade ne s'atténuent pas prématurément de façon marquée ${ }^{48}$ ». Cela ne témoigne évidemment pas, de la part de l'analyste, d'une volonté perverse de maintenir par plaisir l'aiguillon de la souffrance: c'est lié au fait que l'analyse est un processus qui suppose que la volonté d'auto-transformation soit constamment maintenue vivante chez le sujet parce que cette volonté de changer et de se libérer est elle-même la condition pour que l'effort de réflexion et de critique puisse être soutenu. Or la cure étant tout entière une démarche à la fois réflexive et critique dont l'analysant est à la fois le sujet et l'objet, elle n'a de chance de succès qu'à la condition que l'analysant puisse maintenir actif et vivant son effort à la fois réflexif et critique, c'est-à-dire son intérêt pour la transformation de lui-même et sa propre libération. Il faut que cet intérêt pour la transformation émancipatrice soit entretenu si l'on veut que la critique puisse accomplir son œuvre de dissolution des résistances et des obstacles, et que la réflexion permette au sujet l'accès aux pans de sa biographie, de son histoire et de son identité avec lesquels il a perdu le contact. On comprend dès lors que Habermas puisse comprendre et interpréter la psychanalyse comme l'exemple paradigmatique d'une démarche à la fois réflexive, critique et pratique:

c'est le mouvement de la réflexion qui transforme un état en un autre - c'est l'effort émancipatoire de la critique qui transforme l'état pathologique de la compulsion et de l'illusion sur soi-même en celui du conflit dépassé et de la réconciliation avec le langage excommunié (exkommuniziert $)^{49}$.

Toutefois, si la psychanalyse est considérée par Habermas comme paradigmatique dans la manière dont elle articule la réflexion, la critique et l'intérêt émancipatoire, c'est relativement à la théorie critique de la société, au sens où ce que la psychanalyse réussit à accomplir au niveau individuel la théorie critique devrait être capable de l'accomplir au niveau de la société dans son ensemble ${ }^{50}$. Ainsi, lorsque, au sujet de la psychanalyse, Habermas écrit ceci: «l'expérience de la réflexion induite par l'éclaircissement (durch Aufklärung) est l'acte par lequel le sujet se libère précisément d'une situation où il était

48. Freud, «Les voies nouvelles de la thérapeutique psychanalytique», in: De la technique psychanalytique, trad. A. Berman, Paris, PUF, I953, p. I35.

49. CI, p. 277; EI, p. 300. "Exkommuniziert» est à entendre au sens de «soustrait à la communication».

50. Sur les enjeux, les problèmes et les difficultés propres à la façon dont Habermas se réfère à la psychanalyse dans Connaissance et intérêt, on lira Robin Celikates, "La psychanalyse, modèle pour la Théorie critique? Retour sur Connaissance et intérêt de Habermas ", in: Illusio, $\mathrm{n}^{\circ}$ I4-I 5 , Théorie critique de la crise, vol. 3, Dossier "Théorie critique et psychanalyse: altération ", février 2016. 
devenu un objet pour lui-même ${ }^{51}$ ", on imagine sans trop de peine ce qui pourrait en être l'équivalent à l'échelle d'un fonctionnement social. Une société qui a perdu le contrôle conscient de processus aussi déterminants que, par exemple, la division du travail social en son sein, est manifestement une société dont on peut dire qu'elle se trouve dans une situation où elle est devenue un objet pour elle-même: c'est une société parcourue et traversée de processus qui apparaissent comme objectifs précisément parce qu'ils ne sont pas maîtrisés et échappent à tout projet d'organisation rationnelle. On comprend dès lors que le projet puisse être celui de "développer l'idée de base de la psychanalyse dans le sens d'une théorie de la société52 ", d'autant qu'un tel projet n'a précisément pas été mené à bien par Freud luimême selon Habermas.

L'exemple que nous venons de prendre d'une division du travail social apparaissant comme une objectivité non maîtrisée au sein même de la vie sociale n'est précisément pas le genre d'exemple que pourrait prendre Freud parce que son point de vue s'enracine dans des forces naturelles, libidinales et pulsionnelles qui déterminent la société, l'espèce humaine et leur développement historique pour ainsi dire de l'extérieur et à un niveau de profondeur qui n'est pas celui où la société et l'espèce humaine elle-même se constituent en tant que sujets. C'est là, selon Habermas, toute la différence entre Freud et Marx: Marx, quant à lui, «n'a jamais été tenté de dissocier la dynamique de l'évolution et l'activité de l'espèce comme sujet ${ }^{53}$ ", une dissociation à laquelle procède en revanche Freud dès lors que, pour lui, "les forces pulsionnelles libidinales et agressives - puissances préhistoriques de l'évolution, s'emparent en quelque sorte de l'espèce en tant que sujet et déterminent son histoire ${ }^{54}$ ». Tout se passe comme si Freud se donnait d'emblée, au niveau social et collectif, un sujet tout fait, l'espèce humaine avec ses pulsions fondamentales: mais c'est oublier qu'un tel sujet social et collectif — l'espèce humaine comme sujet de sa propre histoire - n'est pas quelque chose de donné d'emblée et que, au contraire, ce qu'on appelle l'histoire est justement le processus même de son autoconstitution et de son autoconservation comme sujet. Comme le rappelle Habermas, c'est depuis ce sujet (l'espèce humaine) constitué comme sujet historique que nous pouvons rétrospectivement tenter de reconstituer, comme le fait Freud, quelles ont été les conditions de «la conservation de l'espèce pour la préhistoire animale de l'espèce humaine». Le point de départ doit donc être pris, selon Habermas, dans l'activité de l'espèce, c'est-à-dire dans l'activité que l'espèce déploie historiquement pour se constituer elle-même comme sujet: c'est bien ce que Marx a fait, contrairement à Freud qui situe le principe dynamique de l'évolution

51. CI, p. 280 (trad. modifiée); EI, p. 302.

52. CI, p. 316 ; EI, p. 346.

53. Ibid.

54. Ibid. 
hors de l'activité humaine et le place dans un fond pulsionnel naturel. Mais si Marx a bien su, quant à lui, inscrire le principe dynamique d'évolution au sein même de l'activité humaine et non en dehors d'elle, la limite de son paradigme est en revanche, selon Habermas, d'avoir réduit l'activité de l'espèce au seul travail, et donc à l'activité instrumentale visant la maîtrise de la nature et de ses forces, et d'avoir en conséquence passé par pertes et profits un autre type d'activité qui joue au contraire un rôle clé chez Freud, à savoir l'activité communicationnelle visant à l'intercompréhension sans contrainte. Et on a pu voir en effet le rôle clé que joue cette activité dans l'analyse puisqu'il s'agit pour un sujet, au moyen de la réflexion critique engagée dans et par la cure, de rétablir la communication avec lui-même ou, du moins, avec les parties de lui-même et de son histoire que leur retrait de la communication lui a rendu incompréhensibles.

Dès lors, la proposition de Habermas est, d'une part, de conserver de Marx l'idée d'autoconstitution et d'autoconservation de l'espèce comme sujet dans et par le déploiement historique de son activité propre (mais sans réduire celle-ci au seul travail et en l'étendant au contraire à l'activité communicationnelle), et, d'autre part, de retenir de Freud l'idée d'une clarification au moyen de la réflexion et d'une dissolution au moyen de la critique des résistances et des obstacles qui s'opposent à l'intercompréhension mutuelle et la communication sans contrainte (mais sans réduire ces résistances et ces obstacles à la seule vie psychique du sujet individuel et en les étendant au contraire à la vie sociale). C'est alors seulement qu'il devient possible pour Habermas d'affirmer que

l'on retrouve dans la société ce qu'on trouve dans la situation clinique: en même temps que la compulsion pathologique elle-même, est posé l'intérêt pour sa suppression; la pathologie des institutions sociales, comme celle de la conscience individuelle, réside dans le milieu du langage et de l'activité communicationnelle, et prend la forme d'une déformation (Verzerrung) structurelle de la communication ${ }^{55}$.

Dans la vie sociale comme dans la vie psychique, c'est l'expérience de la pathologie qui pousse à l'autoréflexion et qui fait naître l'intérêt à se libérer de cette pathologie et à accéder à une forme de vie - en l'occurrence une forme sociale de vie - qui soit libérée des expériences de la mutilation, de la contrainte et de la domination: simplement, dans ce cas, la théorie sociale critique prend le relai de l'analyse, et c'est elle qui devient l'instance porteuse de l'autoréflexion et de la clarification critique, aussi bien en ce que ses représentants (les théoriciens sociaux critiques) pratiquent eux-mêmes cette autoréflexion qu'en ce qu'ils cherchent à la diffuser auprès des autres acteurs sociaux. Comme praticiens de l'activité d'autoréflexion, les théoriciens critiques ont pour tâche de produire une connaissance du social et, plus parti-

55. CI, p. 3I9; EI, p. 349-350. 
culièrement, une connaissance de ce qui, dans le social, prend la forme "d'une objectivation dont le pouvoir (Gewalt) repose uniquement sur le fait que [les acteurs] ne se reconnaisse[nt] pas en elle $^{56}{ }$. Et cette connaissance produite par les théoriciens critiques a sinon directement pour effet, du moins pour intention, de faire naître chez ces mêmes acteurs un «intérêt pour la connaissance, c'est-à-dire l'intérêt qu'ils ont à s'émanciper de ce même pouvoir ${ }^{57}$ » exercé sur eux par des objectivités sociales dont le contrôle leur échappe parce qu'ils ne s'y reconnaissent pas eux-mêmes.

56. CI, p. 3 I 8 ; EI, p. 348.

57. Ibid. 